A skype-based workshop was convened for all relevant parties. In advance, attendees reviewed concerns using the 6 self-guided CCaRM headings, in line with the original CCaRM, as follows:

Having a good circle of support

Having a good shared understanding

Having clear problem areas thought about

Social Participation and Living a Good Life

Keeping people safe and well

Making progress

For each theme, areas of strengths, concerns, and possible fresh approaches were explored. Subsequently, collaborative care plans were refreshed accordingly.

Result. There were 8 such workshops conducted in first PDSA cycle . Participants included support staff and family members, though no service users in this period. All gave positive feedback: that the experience helped with understanding and confidence in roles, and generated fresh ideas to try.

Conclusion.

- This approach helped counter the impact of lockdown in a critical area.

- It was interesting to adapt the CCaRM framework to the online environment.

- The perforce simplification seemed to improve the engagement of carers

- Further work needs to explore potential service user involvement also, and to evaluate the approach longer term.

\section{Supporting mental health during the COVID-19 pandemic: implementation of an e-guide}

Athanasios Hassoulas ${ }^{1 \star}$, Eliana Panayiotou ${ }^{2}$, Srinjay Mukhopadhyay ${ }^{1}$, Ravanth Baskaran ${ }^{1}$ and Nan Zhang ${ }^{1}$

${ }^{1}$ Cardiff University School of Medicine and ${ }^{2}$ Swansea Bay University Health Board

${ }^{\star}$ Corresponding author.

doi: 10.1192/bjo.2021.517

Aims. The COVID-19 pandemic has caused significant disruption to activities of daily living, which in turn has had a profound impact on the mental wellbeing of the public. An e-guide was designed to provide remote support to the general public through the application of a Behavioural Activation approach. Interactive, brief evidencebased exercises were included in the e-guide, along with mood ratings after each exercise to assess any improvements observed.

Method. The e-guide was designed using the Xerte On- Line Toolkits open source software. Videos and interactive exercises were embedded within the resource, forming part of the brief intervention based on cognitivist and behaviourist principles. Information and further support was also provided for young people and parents. Videos from the public highlighting their experiences during the pandemic were also sourced and included (with consent). A pilot was launched to assess the impact of the e-guide. Participants were recruited from Cardiff University, mental health services and a local charity.

Result. The e-guide was piloted on a sample of volunteers $(n=3)$, who completed a brief survey after engaging with the resource. Following the results of the pilot, the e-guide was promoted by the university's marketing team and made available to the public. At the 6-month mark, the e-guide had been accessed by 3228 individuals throughout the UK.
Conclusion. The e-guide has since been disseminated by support services for young people, places of employment and eduction institutions. The national impact of the e-guide is evidenced from the number of people accessing the resource exceeding 3000. With the long-term effects of the pandemic taking hold, it remains crucial to support the wellbeing of the general public through such initiatives that are administered remotely

A quality improvement project to improve the number of section 136 GP discharge summaries from the place of safety

Jemma Hazan ${ }^{1 \star}$, Mikail Ozer², Yathooshan Ramesh ${ }^{2}$ and Richard Westmoreland ${ }^{2}$

${ }^{1}$ Camden and Islington Foundation NHS Trust, Barnet Enfield and Haringey MH NHS Trust and ${ }^{2}$ Barnet Enfield and Haringey MH NHS Trust

${ }^{\star}$ Corresponding author.

doi: $10.1192 /$ bjo.2021.518

Aims. A Quality Improvement project with the aim to increase the number of patients discharged with a GP discharge summary from the Chase Farm Place of Safety over a 12 month time period by $50 \%$. Background. An initial audit was conducted at Chase Farm Place of Safety (POS) to see if patients held under Section 136 of the Mental Health Act (S136) and then discharged home had a GP discharge letter completed and sent. The audit revealed that $0.02 \%$ of patients who were under S136 and discharged home did have a discharge letter sent to the GP.

As a result of the initial audit, key stakeholders were contacted, and involved in the intervention design and implementation. The intervention was introduced and all doctors working in the trust were emailed the new protocol

Method. We implemented the following intervention:

If a patient was registered at a GP Practice then the nursing staff in the POS copied the entry of the discharging doctor from the electronic progress notes and pasted this in to the S136 discharge template on the electronic progress notes and this was emailed to the GP.

We informed Doctors to be aware that their entry would go out to the GP and should contain the following: Impression, Outcome/ Plan, Specific Risk /Safeguarding concerns and specific management plans.

Result. In the initial audit the notes of all patients discharged from the POS under S136 were reviewed over a 3 month period between November and January 2018. We found that 2 out of 89 patients $(0.02 \%)$ had a completed GP summary which was emailed to the GP Practice.

After the intervention was introduced the notes were audited between July and September 2019. We found 33 out of 60 patients (55\%) had a completed GP summary which was emailed to the GP Practice.

Conclusion. There was an improvement of $54.8 \%$ in the number of discharge summaries. Further consideration needs to be given to improving this percentage and understanding what remaining barriers there are.

A quality improvement project to improve handover in the integrated assessment liaison team

Jemma Hazan ${ }^{1 \star}$ and Kirtana Vallabhaneni ${ }^{2}$

${ }^{1}$ Camden and Islington Foundation NHS Trust and ${ }^{2}$ Whittington

Health NHS Trust

${ }^{*}$ Corresponding author.

doi: 10.1192/bjo.2021.519 